\title{
There are no black holes: Pseudo-Complex General Relativity; From Einstein to Zweistein
}

\author{
Walter Greiner*t \\ Frankfurt Institute for Advanced Studies, Frankfurt am Main, Germany \\ E-mail: greinerefias.uni-frankfurt.de
}

\begin{abstract}
The pseudo-complex General Relativity, which includes a minimal length, is resumed and similarities to other theories are pointed out. For a zero minimal length the theory exhibits a remnant contribution, which is associated to a dark energy. It suggests the principle that a mass not only curves the space around it but also changes the vacuum properties, which in turn affect the metric. Some experimental predictions are shortly mentioned. The consequences of the presence of a dark energy are investigated for the case of neutron stars. It is shown that large masses of neutron stars are possible.
\end{abstract}

Frontiers of Fundamental Physics 14

15-18 July 2014

Aix Marseille University (AMU) Saint-Charles Campus, Marseille, France

\footnotetext{
* Speaker.

$\dagger$ In collaboration with P. O. Hess (UNAM, Mexico City) and I. Rodríguez (FIAS)
} 


\section{Introduction}

The theory of General Relativity (GR) has defied up to now all experimental tests (Will 2004) and is considered as the favored theory for gravitation. GR also predicts the existence of socalled black holes, the result of the collapse of a large mass, forming an event horizon. Large mass concentrations of up to several billion solar masses are observed at the center of nearly each galaxy. Nevertheless, up to now no black hole has been seen directly, all observable effects only hint to such objects but do not prove their existence [四]. This might change very soon, namely observations are currently under way [[] to resolve in angular size the so-called black hole, of about four million solar masses, at the center of our galaxy (at the constellation Sgr.A*). Recently, a gas cloud has been detected [ [B], which made contact with the object in Sgr.A* in the second half of 2013. Part of this cloud will aggregate around the central object, increasing significantly its activity. Thus, there is a unique historical chance to study directly this object and its surroundings.

Though GR seems to work very well, there are still several philosophical and practical problems. For example, no experimental tests for very strong gravitational fields have been performed yet, as they appear near to a so-called black hole. Also, as M. Born pointed out [四, 回], there are inconsistencies with Quantum Mechanics, where coordinates and momenta are treated on the same level (they can be interchanged by a canonical transformation). In GR the dominant degrees of freedom appear in the length element square which only depends on coordinates. This leads to the proposal of a modified length element, adding a dependence on the momentum variables. As a consequence, a minimal length appears [6]. There exist other reasons to search for extensions of GR, as demonstrated A. Einstein [ [ $[$, B], whose interest was to unify GR with Electrodynamics. Another serious problem, at least in our opinion, is the existence of an event horizon. From inside this horizon not even light can escape and this inner region will be always excluded to the access of an outside observer at a save distance (an infalling astronaut reaches the internal region but can not send any information about him to the exterior). In our understanding of the universe we feel very uneasy about the appearance of an event horizon. It is probably the reason to study Gavastars [Q⿴囗⿰丿㇄口, [0], which do not have such a horizon. This justifies our interest to search for an alternative extension which eliminates the event horizon, though the object of large mass should resemble in many aspects a black hole.

There exist several attempts to extend GR. We mentioned already M. Born, E. R. Caianiello

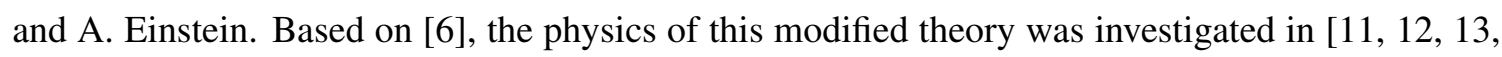

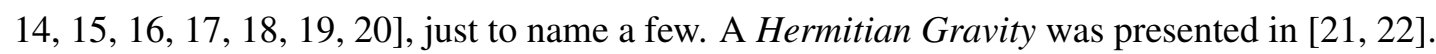

We also proposed an extension of GR to the pseudo-complex General Relativity (pc-GR) [23], [24]. It belongs to an algebraic extension to an 8-dimensional space, introducing pseudo-complex (pc) coordinates. In this contribution the pc-GR is reviewed and the basic concepts are presented. The pc-GR also provides a minimal length and it is shown that all theories mentioned before are contained as a special limit. The theory includes an additional contribution to the right hand side of the Einstein equations, which we denote as a dark energy. The main philosophical change to GR is that in pc-GR a mass not only curves its space around it but also changes the vacuum properties, which in turn effects the metric. The vacuum properties are modeled through the contribution of an external energy-momentum tensor. In the absence of a quantized GR this is the best one can do. (An alternative is to use semi-classical Quantum Mechanics, which assumed a fixed background metric. 
However, due to that no influence of a dark energy distribution on the metric can be calculated and the event horizon remains.)

Within this contribution we use the signature $(+---)$ for the metric.

\section{The pseudo-complex General Relativity}

In pc-Gr the real coordinates are algebraically extended to pseudo-complex coordinates

$$
x^{\mu} \rightarrow X^{\mu}=x^{\mu}+I y^{\mu}
$$

with $I^{2}=1$, which is the reason to call it pseudo-complex. The $x^{\mu}$ are the usual coordinates in GR and $y^{\mu}$ are the pseudo-imaginary components. The set $\left(x^{\mu}, y^{\mu}\right)$ defines the coordinates of a manifold. An alternative way to express these coordinates is to introduce $\sigma_{ \pm}=\frac{1}{2}(1 \pm I)$, which leads to an equivalent representation, namely

$$
\begin{aligned}
& X^{\mu}=X_{+}^{\mu} \sigma_{+}+X_{-}^{\mu} \sigma_{-} \\
& X_{ \pm}^{\mu}=x^{\mu} \pm y^{\mu} .
\end{aligned}
$$

It is easy to verify that $\sigma_{ \pm}^{2}=\sigma_{ \pm}$and $\sigma_{+} \sigma_{-}=0$. These properties show that when an element proportional to $\sigma_{+}$is multiplied with another one proportional to $\sigma_{-}$, the product is zero, which implies the existence of a zero-divisor. In other words, the pc-variables do not form a field but rather a ring. Mathematical manipulations can be done in each zero divisor component, independently to the other one. For example, using arbitrary functions

$$
F(X)=F\left(X_{+}\right) \sigma_{+}+F\left(X_{-}\right) \sigma_{-}
$$

(the same for $G(X)$ ) the product of both is given by

$$
\begin{gathered}
F(X) G(X) \\
= \\
F\left(X_{+}\right) G\left(X_{+}\right) \sigma_{+}+F\left(X_{-}\right) G\left(X_{-}\right) \sigma_{-} .
\end{gathered}
$$

The mathematics is very similar to the complex variables and details can be found in [25, 26]. An important property is that any element, which is proportional to only $\sigma_{+}$or $\sigma_{-}$has zero norm, defining the complex conjugate by inverting $I$ to $-I$ and the norm of $X$ through

$$
|X|=X X^{*}
$$

This can be seen as follows: Under complex conjugation we have $\sigma_{ \pm}^{*}=\sigma_{\mp}$. An element within the zero-divisor is, for example $\lambda \sigma_{-}$, with $\lambda$ being an arbitrary function. Its pseudo-complex conjugate is $\lambda \sigma_{+}$and a product with the former one is zero due to $\sigma_{-} \sigma_{+}=0$. 
These features lead to the proposition to define a GR in each zero-divisor component [22], 24], with the pseudo-complex metric

$$
g_{\mu v}(X)=g_{\mu v}^{+}\left(X_{+}\right) \sigma_{+}+g^{-}\left(X_{-}\right) \sigma_{-} .
$$

The metric functions $g_{\mu v}^{ \pm}\left(X_{ \pm}\right)$are obtained by varying the standard action of GR, namely $S_{ \pm}=\sqrt{-g_{ \pm}} \mathscr{R}_{ \pm}$, with $g_{ \pm}$the determinant of the metric $g_{\mu \mu}^{ \pm}$and $\mathscr{R}_{ \pm}$the Riemann scalar in each component. However, using the standard condition for the variation, i.e., $\delta S=\delta S_{+} \sigma_{+}+\delta S_{-} \sigma_{-}=$ 0 , leads to $\delta S_{ \pm}=0$, which describes two independent theories of GR. In order to obtain a new theory, it was proposed in [27, 28] (in the context of field theories) to use a modified variational principle, namely

$$
\delta S=\delta S_{+} \sigma_{+}+\delta S_{-} \sigma_{-}=\lambda \sigma_{ \pm}
$$

i.e., it is proportional to either $\sigma_{+}$or $\sigma_{-}$. Such elements are within the zero-divisor and have zero norm, which justifies the denomination of a generalized zeros. This modification is equivalent to add in $S_{-}$an additional term, whose variation just gives $-\lambda \sigma_{-}$. The Einstein equations are changed to

$$
\mathscr{R}_{\mu v}-\frac{1}{2} g_{\mu v}(X) \mathscr{R}=-\frac{16 \pi \kappa}{c^{2}} T_{\mu v}^{\Lambda} \sigma_{-}
$$

(When projected to the real-part, the $\sigma_{-}$on the left hand side is mapped to $\frac{1}{2}$, reducing the factor 16 to 8.) The right hand side is a consequence of the modified variational principle. The convention is to set the contribution proportional to $\sigma_{-}$and equivalent results are obtained when $\sigma_{+}$is taken instead. The index $\Lambda$ in the energy-momentum tensor indicates that we interpret it as the contribution of a dark energy. This term is equivalently obtained when one assumes that the mass not only curves the space but also changes the vacuum properties in its neighborhood, for which a model is proposed. That mass changes the vacuum structure is not new and discussed in [29], where a semi-classical approach in Quantum Mechanics is applied (fixed background metric) in the calculation of vacuum fluctuations. With this procedure, the vacuum fluctuations can be determined but not their influence on the metric. Contrary to that, when the vacuum fluctuations are included via a model for $T_{\mu \nu}^{\Lambda}$, as we do it, one obtains the modified metric.

Quite often, $y^{\mu}$ is identified with $\left(\frac{l}{c}\right) u^{\mu}$ [四, [1, 目], where $c$ is the light velocity, $u^{\mu}$ the components of the 4-velocity and $l$ has the units of length, interpreted to be a minimal length of the theory. The factor $\left(\frac{l}{c}\right)$ is introduced for dimensional reasons. Thus, the minimal length is a parameter of the theory and, therefore, is not affected by any Lorentz transformation, which demonstrates that theories with a minimal length not necessarily imply a violation of the Lorentz symmetry. However, one has to be careful with the interpretation of $y^{\mu}$, because in case to be simply proportional to the components of the 4-velocity, the $y^{\mu}$ is rather in the tangent space of the manifold with coordinates $\left(x^{\mu}\right)$. A parallel transport in this combined manifold- tangent space is quite complicated [ए], [2, [3]]! The problem gets easier to handle when the space is flat. Then, the $x^{\mu}$ and 
$u^{\mu}$ transform equally. Insisting that $\left(x^{\mu}, y^{\mu}\right)$ are still the coordinates of a pseudo-complex manifold, the relation of $y^{\mu}$ to the 4-velocity components has to be more involved. Nevertheless, in order to relate the theory to existing models with a minimal length, we keep the interpretation of the pseudo-imaginary components being $\frac{l}{c} u^{\mu}$. When the contributions from the pseudo-imaginary component are too small, $l$ (or $y^{\mu}$ ) can simply be set to zero. What remains is the extra contribution of the dark energy, due to the modified variational principle.

When the minimal length is assumed to be very small but still different from zero (for example the size of a Planck length) and the same functional form of the metrics in the zero-divisor components are chosen $\left(g_{\mu \mu}^{+}\left(X_{+}\right)=g_{\mu \nu}\left(X_{+}\right)\right.$and $\left.g_{\mu \mu}^{-}\left(X_{-}\right)=g_{\mu v}\left(X_{-}\right)\right)$, the pseudo-complex length element square

$$
d \omega^{2}=g_{\mu v}(X) d X^{\mu} d X^{v}
$$

simplifies further: First, the metric functions $g_{\mu v}\left(X_{ \pm}\right)$are expanded in $\left(\frac{l}{c}\right) u^{\mu}$. Considering that $\left|u^{\mu}\right| \leq c$ and $l$ of the order of the Planck length, thus $\left(\frac{l}{c}\right)\left|u^{\mu}\right|<<1$, it is sufficient to restrict to the first term in the expansion, i.e., $g_{\mu v}\left(X_{ \pm}\right) \approx g_{\mu v}(x)$. The situation is different expanding in $d X^{\mu}=d x^{\mu}+\left(\frac{l}{c}\right) d u^{\mu}$, due to the appearance of $d u^{\mu}$, which is an acceleration and may reach values proportional to $1 / l[$ [6].

Expanding (2. ), with the approximations just given, the length element square acquires the form

$$
\begin{aligned}
d \omega^{2} \approx & g_{\mu v}(x)\left[d x^{\mu} d x^{v}+\left(\frac{l}{c}\right)^{2} d u^{\mu} d u^{v}\right] \\
& +2 \operatorname{Ig}_{\mu v}(x) d x^{\mu} d y^{v} .
\end{aligned}
$$

The $d \omega^{2}$ is required to be real, which implies that the pseudo-imaginary component is zero, or

$$
g_{\mu v}(x) d x^{\mu} d y^{v}=0
$$

which is nothing but the differential form of the dispersion relation:

$$
\begin{aligned}
d\left[g_{\mu v} u^{\mu} u^{v}\right] & =g_{\mu v} u^{\mu} d u^{v} \\
& =2 g_{\mu v} \frac{d x^{\mu}}{d \tau} d u^{\mu},
\end{aligned}
$$

where the symmetric nature of the metric has been used in the last step and $\tau$ is the eigen-time. Note, that the dispersion relation is not implemented by hand, as usually done, but is a consequence of demanding a real length element square.

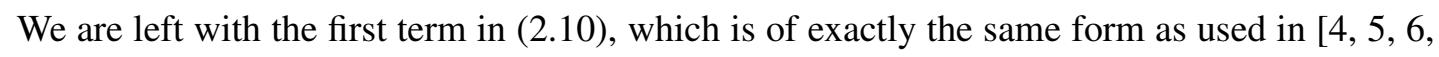
[1], [7]]. Thus, all these theories are particular cases of pc-GR.

In what follows, the effects due to the minimal length and the acceleration are negligible and therefore the lowest order contributions due $l$ are skipped. A study of contributions and its size due 
to $l$ are important from a conceptual point of view. The length element square $d \omega^{2}$ reduces now to the standard form in GR, but the modified variational principle still is responsible for the dark energy contribution.

\section{Features and Predictions}

A particular feature of pc-GR is the disappearance of the event horizon. In the pc-Schwarzschild solution, the $g_{00}$ component has the form [231, [24]

$$
\begin{aligned}
g_{00} & =1-\frac{2 m}{r}+\frac{B}{2 r^{3}} \\
B & =b m^{3} .
\end{aligned}
$$

The $B$ is a measure of the coupling of the mass to the dark energy and depends on $m=\frac{4 \pi \kappa}{c^{2}} M$ ( $\kappa$ is the gravitational constant), with $M$ as the mass of the central object. In order that there is no event horizon, the value of $b$ is chosen such that $g_{00}>0$ everywhere. This leads to the lower limit for $b$ of $\frac{64}{27}$. The $r$-dependence of the correction term is such that no effects can be measured yet within solar system experiments [B]]. The square root of $g_{00}$ is proportional to an effective potential with zero orbital angular momentum [B]]. It has a minimum at approximately two-thirds of the Schwarzschild radius [23] and increases with $B$. This lead in [23] to the interpretation that an infalling test particle is accelerated inwards until it reaches the minimum and toward smaller distances the particle feels a repulsive force. Therefore, this minimum represents a halting point in the collapse of a star, or in different words, it indicates the size of the star. Of course, this is only a rough estimate and the interior of the star has to be considered, too, which leads to the investigation of neutron stars within the pc-GR. For the Kerr metric similar conclusions are drawn [24].

In [32] the circular orbits around the central rotating mass are investigated. The dependence of the orbital frequency on the metric is found to be

$$
\omega_{ \pm}=c \frac{-g_{03}^{\prime} \pm \sqrt{\left(g_{03}^{\prime}\right)^{2}-g_{00}^{\prime} g_{33}^{\prime}}}{g_{33}^{\prime}} .
$$

It depends on the slope of the metric. In standard GR the slope increases towards smaller radial distances and becomes maximal when $g_{00}$ is zero, approximately at the position of the event horizon. This is the reason why the orbital frequency increases continuously, as seen in Fig. W. In pc-GR the slope increases up to a turning point and then is inverted until reaching the minimum at approximately two-thirds of the Schwarzschild radius. At the turning point the orbital frequency has a maximum and for smaller radial distances the frequency decreases again. This result is depicted in Fig. 田.

In [32] also the red-shift was calculated as a function of the radial distance. The red shift is given by

$$
z=\frac{\sqrt{r^{2}+a^{2} \cos ^{2}(\vartheta)}}{\sqrt{r^{2}-2 m r+a^{2} \cos ^{2}(\vartheta)+\frac{B}{2 r}}}-1
$$




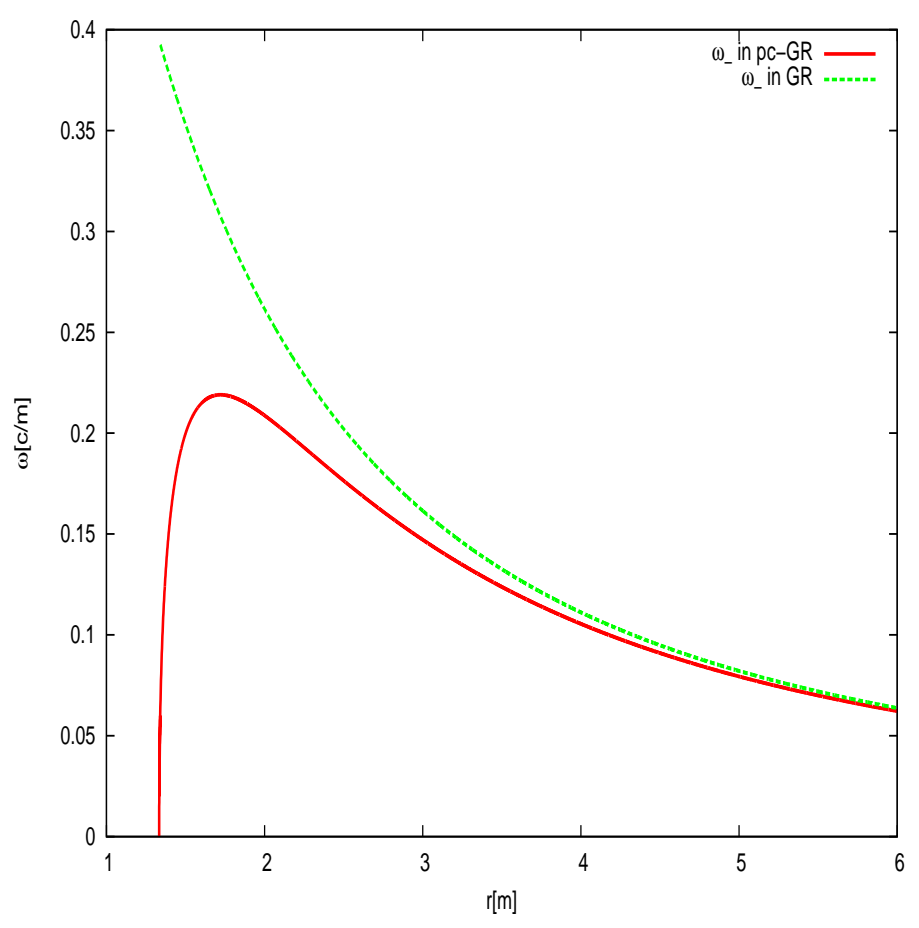

Figure 1: Comparison of the orbital frequency within GR to pc-GR as a function in the radial distance $r$ (in units of $m$ ). The frequency is given in units of $m / c$. A value of $\omega=0.219$ correspond to 9.4 minutes for a mass of about four million solar masses. The rotational Kerr parameter is $a=-0.995$ and $b=64 / 27$.

with the rotational parameter

$$
a=-\frac{\kappa J}{m c^{3}} .
$$

The $J$ defines the angular momentum. For the equatorial plane the $\vartheta=\frac{\pi}{2}$. The red-shift increases towards smaller distances and the curve is shifted to smaller $r$-values, however, never reaching infinity for $b>\frac{64}{27}$.

To test GR and pc-GR, a possibility is to measure the orbital frequency of Quasi Periodic Objects (QPO) and the redshift, deduced from Fe $\mathrm{K} \alpha$ lines emitted by the QPO. In a consistent theory the same radial distance should emerge in both observables. Standard GR and pc-Gr predict a quite different behavior near a large mass object and it should be possible to be measure it.

The findings within pc-GR seem to be in accordance with recent observations (though, if the QPO are in the accretion disk is still debated). With respect to the red-shift, the results within pc-GR also imply that light emitted from a given distance appears brighter in pc-GR than in GR.

Not resumed in this contribution are different possible scenarios of the future of the universe, using the pc-Robertson-Walker model [B3]]. Instead of the big ripp also solutions are possible where the acceleration of the universe approaches a constant value or even zero acceleration. Investigations of an oscillating universe are in process. 


\section{Neutron Stars}

In this contribution, we will concentrate on the general features and applications. For simplicity, from now on the unit for the velocity of light is set to one $(c=1)$. The mass $m$ is measured in $\mathrm{km}$.

Outside the star we have a good estimation how the energy density is coupled to the mass (how it falls off as a function of the radial distance). The condition is that the additional contribution to $g_{00}$ has to be such, that it can not be detected yet in solar system experiments, the most accurate known. Using the Parametrized Post Newton Formalism [B]], it is shown that there is no place for a $1 / r^{2}$ correction. Thus, it has to behave at least as $1 / r^{3}$, which we finally assume. In [20]) the vacuum fluctuation near a Schwarzschild black hole was determined within the Boulware vacuum, using a semi-classical approach to quantum mechanics, i.e. a fixed back ground metric is assumed. As a result, the dark energy density falls off as $1 / r^{6}$, apart from further lower order corrections. In [B2] different radial behaviors were investigated and shown that the main characteristics of the predicted observational data do not change much. In conclusion, the estimated functional dependence of the dark energy density outside the star does not suffer from basic problems. This changes when the inside of a star is considered, because there the coupling of the mass density to the vacuum fluctuations has to be known, which we don't!

Outside the star there is an additional condition to the character of the dark energy fluid: An anisotropic fluid has to be assumed and the argument is as follows. Taking the TOV equation for the in general anisotropic dark energy fluid, we have

$$
\begin{aligned}
\frac{d p_{\Lambda r}}{d r}= & -\frac{\left(\varepsilon_{\Lambda}(r)+p_{\Lambda r}(r)\right)}{r\left[r-2 m+2 m_{\Lambda}(r)\right]} \\
& \times\left[m-m_{\Lambda}(r)+4 \pi k r^{3} p_{\Lambda r}(r)\right] \\
& +\frac{2\left(p_{\Lambda \theta}-p_{\Lambda r}\right)}{r} .
\end{aligned}
$$

The indexes $r$ and $\theta$ refer to the radial and tangential pressure, respectively. We require that the Null Dominant Energy Condition [34] is satisfied. In many cases some of the energy conditions are not satisfied [BS] . For example, negative energy densities are possible, which violate the Weak energy condition (WEC). The NDEC is less stringent and reads

$$
T_{\mu v} l^{\mu} l^{\nu} \geq 0
$$

where $l^{\mu}$ are the components of a null-vector, and

$$
W^{\mu}=T_{v}^{\mu} l^{v}
$$

is a non-space like vector. This leads to the conditions

$$
\begin{aligned}
\varepsilon_{\Lambda}+p_{\Lambda r} & \geq 0 \\
\left|\varepsilon_{\Lambda}\right| & \geq\left|p_{\Lambda r}\right|
\end{aligned}
$$


which are satisfied also for a negative energy density and the equation of state $p=-\varepsilon_{\Lambda}$. But with this equation of state, the first term in (4. (لل ) vanishes. When an isotropic fluid is assumed $\left(p_{\theta}=p_{r}\right)$, the radial derivative of the pressure is zero, therefore the pressure is constant and also the energydensity. Thus, in order to allow a fall-off of the energy density, an anisotropic fluid has to be assumed.

Inside the star, we take for simplicity a model with an isotropic fluid for both the baryonic and the dark energy density. Note, that the property of the dark energy density changes between the inside and outside region of the star. For the baryonic equation of state we use the one as deduced in [B6], within the hadronic chiral $S U(3)$ model, which also is equivalent to an isotropic fluid. It is a mean field theory and is able to reproduce the binding energy for a large range of nuclei. Because the coupling between mass and the dark energy is not known, we rely on models. As in [37], we assume a linear coupling, i.e., $\varepsilon_{\Lambda}=\alpha \varepsilon_{m}$, with a negative $\alpha$.

In Fig $\square$ the total mass versus its radius is plotted. The stable branch is represented by the part with a positive slope of the curve. As can be see, a mass of up to six solar masses is easily achieved. Larger masses are not obtained due to the following mechanism: When $\alpha$ is too large, the fall-off of the dark energy is not strong enough for increasing $r$ and its repulsive effect wins over the gravitational attraction. The outer layers of the star are evaporated as a consequence. The problem lies clearly with the right coupling of the dark-energy to the mass. A different dependence than the linear one has to be assumed, which is to be reconsidered in future.

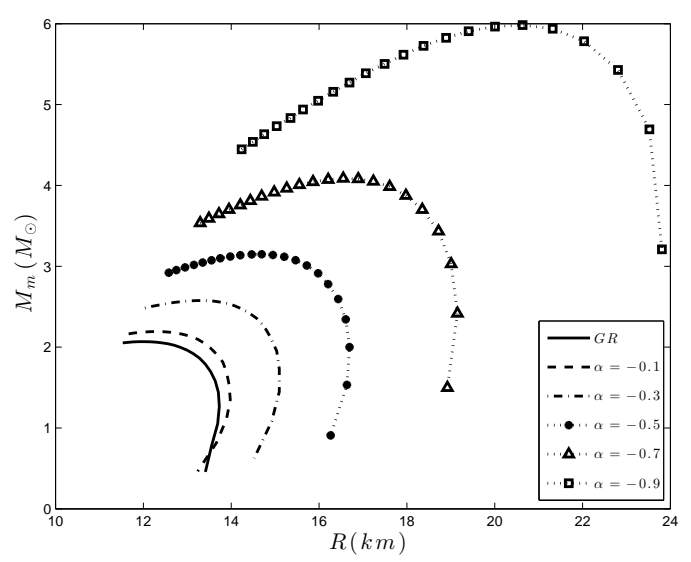

Figure 2: The baryonic total mass versus total radius for different values of the coefficient $\alpha$. The central $\Lambda$-term pressure $p_{\Lambda c}$ has been fixed to $1 \varepsilon_{0}$, where $\varepsilon_{0}$ is the nuclear energy density.

Another interesting observation is that for six solar masses the Schwarzschild radius of possible stars range from $14 \mathrm{~km}$ to $24 \mathrm{~km}$, i.e. of the same order as the Schwarzschild radius. In Fig. [] the density profile for a star with different values of $\alpha$ is shown. For six solar masses $(\alpha=-0.9)$ the radius is just above the Schwarzschild radius. Thus, it behaves in pc-GR as a near black hole (not really black, because it still may emit light) with a large red shift at the surface. For the next lower value of $\alpha(=-0.7)$ the maximal mass is around four solar masses and the corresponding Schwarzschild radius is about $11.7 \mathrm{~km}$. Therefore, between 4 and 6 solar masses the neutron stars resembles more and more a classical black hole. This seems to be in accordance with observation. 


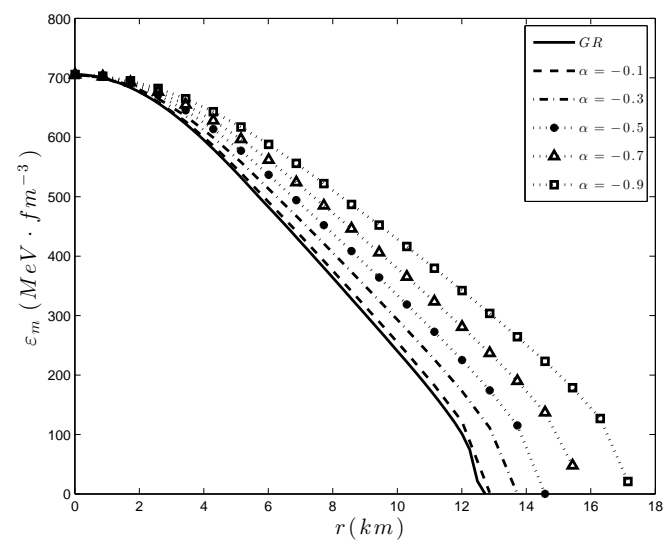

Figure 3: The baryonic energy density profile for different values of the coefficient $\alpha$. The central $\Lambda$-term pressure $p_{\Lambda c}$ has been fixed to $1 \varepsilon_{0}$, where $\varepsilon_{0}$ is the nuclear energy density.

The maximal mass of a neutron star observed is slightly above two solar masses [38] and our theory shows that above this value the neutron star approaches a star resembling a black hole.

\section{Conclusions}

A review of the pseudo-complex Theory of General Relativity was given. It was shown that the theory implies the existence of a minimal length. In the limit of a small length, the pc-GR contains several other theories, which also contain a minimal length parameter. Modifying the variational principle did lead to a contribution of a dark energy-momentum tensor on the right hand side of the Einstein equations. A physical principle emerges from it: Mass not only curves the space but also modifies the vacuum properties around that mass. The dark energy contributions react back on the curvature of space, halting the collapse of any size of mass, and finally the event horizon disappears. For the dark energy contribution a model had to be used, due to the lack of a quantized quantum theory.

We resumed some experimental predictions, neglecting the effects of the minimal length:

i) The radius of the star is smaller than the Schwarzschild radius.

ii) The surface of the star should be seen in future, though, light emitted from the surface is largely red-shifted.

iii) The orbital frequency of a particle in a circular orbit first increases towards smaller distances, until it reaches a maximum and then decreases again.

iv) The red-shift curve is shifted to smaller distances and thus an accretion disk appears brighter.

v) Neutron stars with larger masses are possible, though it is probably doubtful that for large masses nuclear matter is still present. The radii of the massive neutron stars rapidly approach with increasing mass the Schwarzschild radius. These neutron stars resemble more black holes than neutron stars.

\section{References}

[1] M.A. Abramovicz and W. Kluzniak, A \& A 396 (2002), L31. 
[2] VLBA (The Very Long Baseline Array), (2013), http://www.vlba.nrao.edu

[3] S. Gillessen, F. Eisenhauer, T.K. Fritz, H. Bartko, K. Dodds-Eden, T. Ott, R. Genzel, ApJ 707 (2009), 114.

[4] M. Born, Proc. Roy. Soc. A 165 (1938), 291.

[5] M. Born, Rev. Mod. Phys. 21 (1949), 463.

[6] E.R.Caianiello, Nuovo Cim. Lett. 32 (1981), 65.

[7] A. Einstein, Ann. Math. 46 (1945), 578.

[8] A. Einstein, Rev. Mod. Phys. 20 (1948), 35.

[9] P.O. Mazur and E. Mottola, Proc. of the Nation. Acad. Sciences of U.S.A. 101, No. 26 (2001), 9545.

[10] Mazur P.O. and Mottola E., Proceedings of the 6th Workshop on Quantum Field Theory under the Infuence of external Conditions, Univ. Oklahoma, Norman, U.S.A., Sept. 15-19 (2004), 350.

[11] H.E. Brandt, Found. Phys. Lett. 2 (1989), 39.

[12] H.E. Brandt,Found. Phys. Lett. 4 (1989), 523.

[13] H.E. Brandt, Found. Phys. Lett. 6 (1993), 245.

[14] R.G. Beil, Int. J. Theor. Phys. 26 (1987), 189.

[15] R.G. Beil, Int. J. Theor. Phys. 28 (1989), 659.

[16] R.G. Beil, Int. J. Theor. Phys. 31 (1992), 1025.

[17] R.G. Beil, Found. Phys. 33 (2003), 1107.

[18] J.W. Moffat, Phys. Rev. D 19 (1979), 3554.

[19] G. Kunstatter, J.W. Moffat and J. Malzan, J. Math. Phys. 24 (1983), 886.

[20] G. Kunstatter and R. Yates, J. Phys. A 14 (1981), 847.

[21] C. Mantz and T. Prokopec. (2008), [arXiv:gr-qc10804.0213].

[22] C. Mantz and T.Prokopec, Found. Phys. 41 (2011), 1597.

[23] P.O. Hess and W. Greiner,, Int. J. Mod. Phys. E 18 (2009), 51.

[24] G. Caspar, T. Schönenbach, P.O. Hess, M. Schäfer and W. Greiner, Int. J. Mod. Phys. E 21 (2012), 1250015.

[25] F. Antonuccio, Semi-Complex Analysis and Mathematical Physics, (1993) [gr-qc/9311032].

[26] I.L. Kantor and A.S. Solodovnikov, Hypercomplex Numbers. An Elementary Introduction to Algebra, (Springer, Heidelberg,1989)

[27] F.P. Schuller, Dirac-Born-Infeld Kinematics, Maximal Acceleration and Almost Product Manifolds, Ph.D. Thesis, Cambridge, (2003).

[28] F.P. Schuller, Wohlfarth M.N.R. and Grimm T.W.: 2003, Class. Quantum Grav. 20 (2003), 1.

[29] M. Visser, Phys. Rev. D 54 (1996), 5116.

[30] C.M. Will 2004, PRAMANA 63 (2004), 731.

[31] C.W. Misner, K.S. Thorne and J.A. Wheeler, Gravitation, (W. H. Freeman and Company, San Francisco,1973)) 
[32] T. Schönenbach, G. Caspar, P.O. Hess, T. Boller, A. Müller, M. Schäfer and W. Greiner, Month. Notic. of the Roy. Astron. Soc. 430 (2013), 2999.

[33] P.O. Hess, L. Maghlaoui and W. Greiner, Int. J. Mod. Phys. E 19 (2010), 1315.

[34] S. Carroll, Spacetime and Geometry: An introduction to General Relativity, (Addison Wesley, N.Y., 2004).

[35] M. Visser and C. Barceló, (1999), [arXiv:gr-qc/0001099].

[36] V. Dexheimer and S. Schramm, The Astroph. J. 3 (2008), 943.

[37] C.R. Ghezzi, (2009), Astroph. and Space Science. 333 (2011), 437.

[38] J. Antoniadis et al., Science 26, (2013), 340(6131), 1233232. 